\title{
Milling gate vibrations analysis via Hilbert-Huang transform
}

\author{
Grzegorz Litak ${ }^{1, *}$, Marek Iwaniec ${ }^{1}$, and Joanna Iwaniec $^{2}$ \\ ${ }^{1}$ AGH University of Science and Technology, Faculty of Mechanical Engineering and Robotics, Department of Process Control, \\ Mickiewicz Alley 30, 30-059 Krakow, Poland \\ ${ }^{2}$ AGH University of Science and Technology, Faculty of Mechanical Engineering and Robotics, Department of Robotics \\ and Mechatronics, Mickiewicz Alley 30, 30-059 Krakow, Poland
}

\begin{abstract}
The study aimed at identification of the milling gate modal parameters: natural frequencies and mode shapes, in order to establish the working range free from resonant areas. The evaluation was performed under exploitation loads, generated in the course of the milling process. System responses, in the form of the acceleration time histories, were analysed with the application of the Hilbert-Huang method data analysis. Selection of this method was motivated by the fact that it does not require stationarity of the analysed and provides information about nonlinearities in vibration empirical modes. In contrast to classical nonlinear system identification methods, the Hilbert-Huang method does not require measurements of excitations acting on the system of interest nor linear system dynamic behaviour in a broad frequency range around any operating point.
\end{abstract}

\section{Introduction}

Vast majority of real mechanical systems is nonlinear to a certain degree. Nonlinear industrial systems can be classified as systems designed for work in nonlinear ranges of dynamic characteristics and systems of nonlinear properties resulting from fault appearance. Although the sources of nonlinear properties can vary $[1,2,3,4]$, all the nonlinear systems exhibit some common properties. In general, they do not follow the superposition principle and exhibit complex phenomena unusual for linear systems, such as jumps, self-induced and chaotic vibrations, changes in natural frequencies resulting from changes in excitation amplitudes and coexistence of many stable equilibrium positions. In view of these properties, classical identification methods cannot be used for the purposes of nonlinear system identification.

The main emphasis of the paper is put on the experimental identification of modal parameters (natural frequencies and mode shapes) of the milling gate subjected to operational loads resulting from the milling process. For the purposes of experimental data analysis, the Hilbert-Huang transform was used. The choice of this method was motivated by the fact, that it does not require stationarity of the analysed data and, therefore, various phases of milling process can be selected for analysis. Furthermore, it provides information about nonlinearities in vibration empirical modes. On the contrary to the classical nonlinear system identification methods, the Hilbert-Huang method requires neither the measurement of excitations acting on the system of interest nor linear system dynamic behaviour in a broad frequency range around any operating point $[5,6]$. The possibility of system identification in the exploitation conditions is of key importance, since the measurement of operational exciting forces is difficult or impossible to carry out and diagnostics of many industrial systems, for the economical or technological reasons, should be carried out during the normal work [7]. Obtained results made it possible to establish the milling gate work range by avoiding resonant areas.

The structure of the paper is as follows: after short introduction, the measurement setup is presented. Then the consecutive steps of the Hilbert-Huang method application to the measured data are shown. Further part of the paper is devoted to discussion of the obtained results of analysis. Finally, the paper is concluded in the last section.

\section{Experimental standing and measurement results}

In the experiments, the typical milling process of different thickness of the cut layer realised on the milling machine was conducted. The excitation during the experiment was given by operational forces generated during the milling process. The aim of the investigation was identification of the modal frequencies as the function of the amplitude of local measured vibration. Assuming that in the set of the main attention was focused on the analysis of vibration of the gate of milling machines.

In the measurements the 28-channel dynamic analyser SCADAS III was used. The analyser was controlled by TestLab ImpactTesting software by Siemens-LMS. One channel was used for force measurements and 24 channels

* Corresponding author: g.litak@.pollub.pl 
were used for measuring dynamical response in the chosen points of the gate. Additional 3 channels were used for registration the ambient vibrations as the referents points. Dynamical response was measured by the 3D piezoelectric accelerometers PCB 356A16 in the chosen points distributed on the gate.

The view of the experimental set and one of the typical experimental results are presented in Fig. 1.

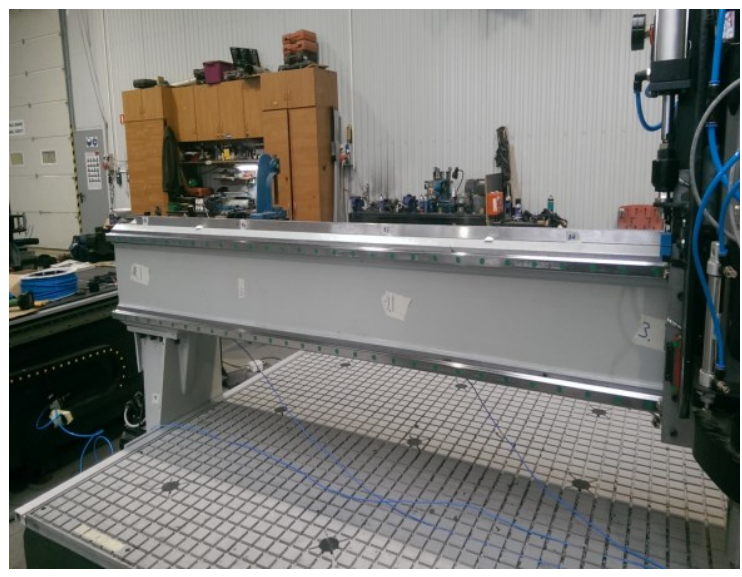

(a)

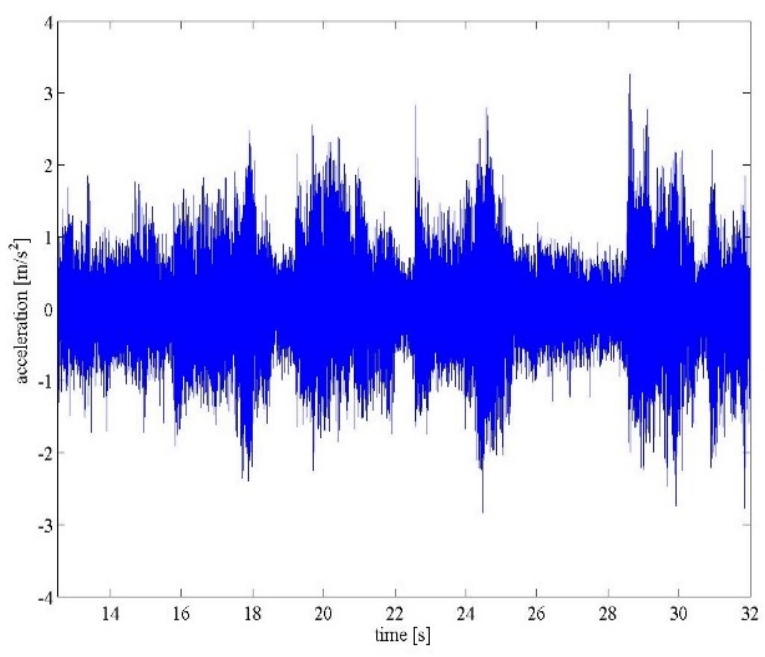

(b)

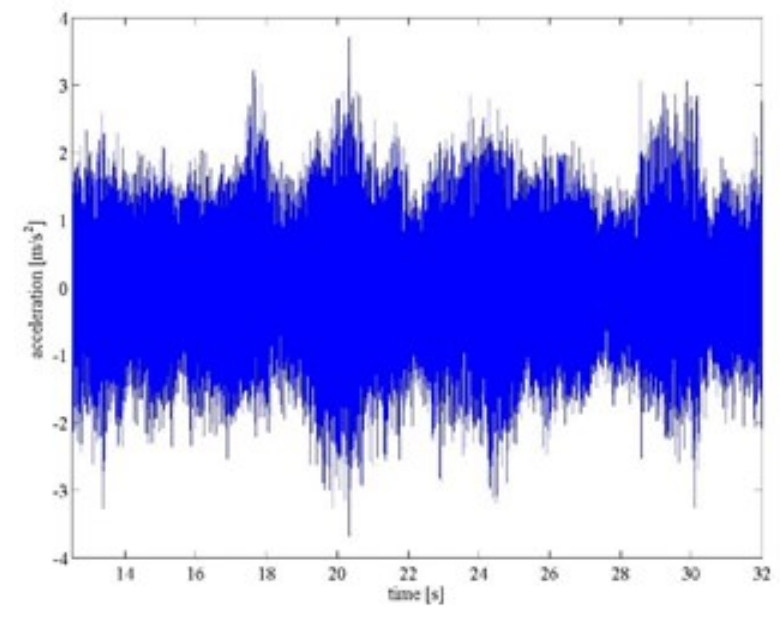

(c)

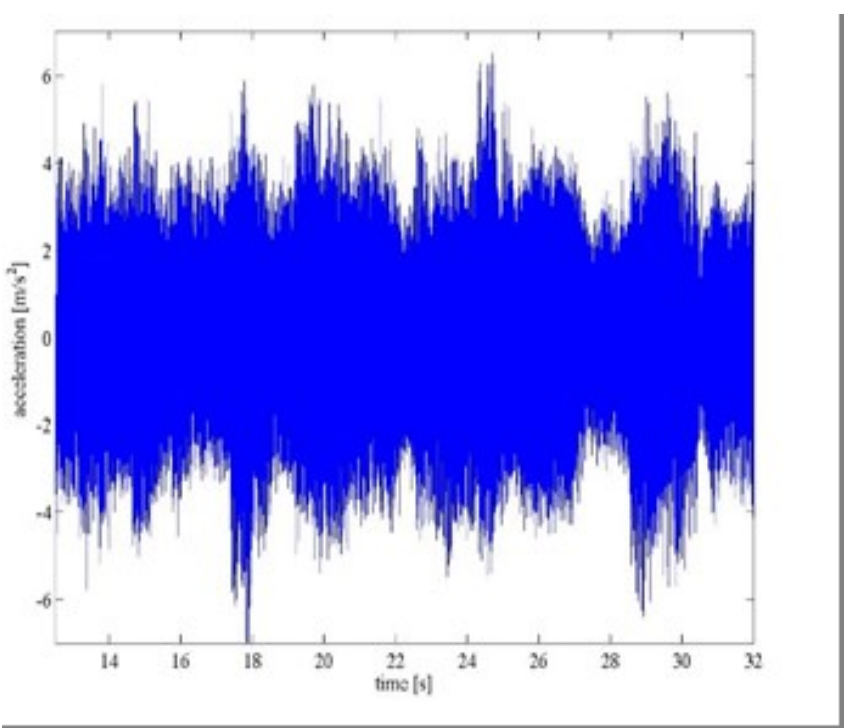

(d)

Fig. 1. Typical layout of a superelastic SMA oscillator. Milling gate (a) and (b) example of the time series measured during milling experiment. From the accelerometer located on milling gate, cases (b) - (d) denote the directions $\mathrm{x}, \mathrm{y}, \mathrm{z}$.

\section{Hilbert-Huang analysis}

Performing the Hilbert-Huang analysis [8,9], the signal is firstly decomposed into the Huang empirical mode (EMD). In our case, the acceleration signal from the accelerometer (the second table point B4 modal analysis structure for fastening the spindle moulder) $A(t)$ (see Fig. 1). Operating frequency of milling was much higher than the sample rate $1024 \mathrm{~Hz}$. In our analysis, we used even less frequency of 102.4 $\mathrm{Hz}$ (when every 10 value) to save the computation time. The main aim was to examine clamping structure vibrations of the milling machine. Obviously, $\boldsymbol{A}(t)$ has three spatial components of $A_{i}$, where $i=x, y, z$, respectively. Figures $1 \mathrm{~b}-\mathrm{d}$ clearly indicate that the predominant direction of the vibration is determined by the $z$ axis. In this direction, the amplitude of the vibration is the largest, and decreases subsequently in the $y$-direction and $x$-directions.

In addition to the above, it is worth paying attention to the phenomenon of intermittency in the presented series. This behaviour may indicate on a non-smooth milling or/and an emergence of the so-called vibration type chatter, with a considerable increase in the amplitude. Each of vector components of the acceleration vector $A(t)$ can be decomposed into the empirical modes $A_{\mathrm{i}}{ }^{1}(t), A_{\mathrm{i}}{ }^{2}(t), \ldots, A_{\mathrm{i}}{ }^{\mathrm{m}}(t)$ [9-11] (Fig. 2a-c).

$$
A_{i}(t)=\sum_{j=1}^{m} A_{i}^{j}(t)+r_{m},
$$

where $r_{\mathrm{m}}$ stands for a truncation error on the $m$ empirical mode. The subsequent $j$ modal time series $A_{\mathrm{i}}{ }^{\mathrm{m}}$ is obtained after subtracting the corresponding means from the maximum and minimum values of the previous mode $\mathrm{rm}^{-1}$ (the envelope of the time series) interpolated by cubic polynomials along the time. From Fig. 2 it is clear, that only the first three have relatively large amplitudes. This is also shown in Tab. 1 


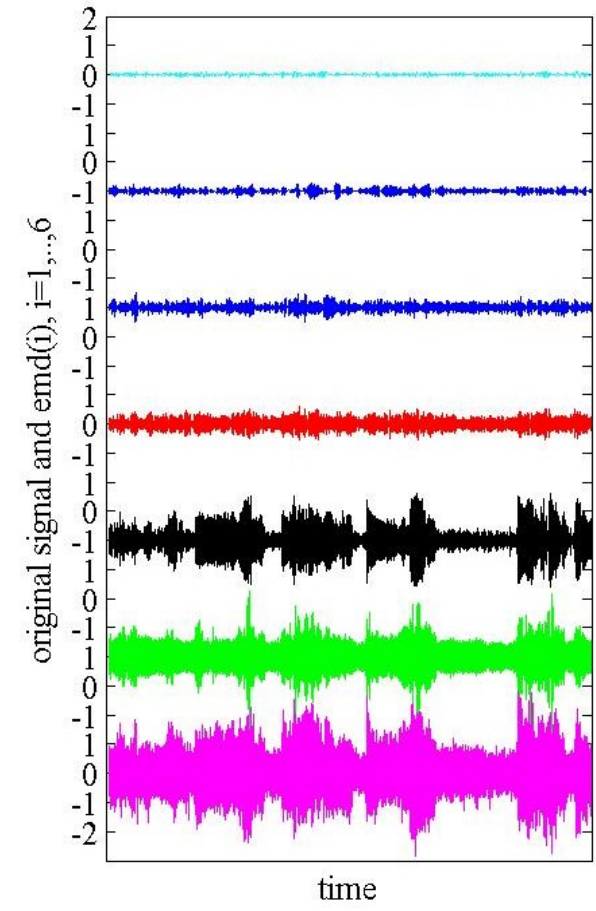

(a)

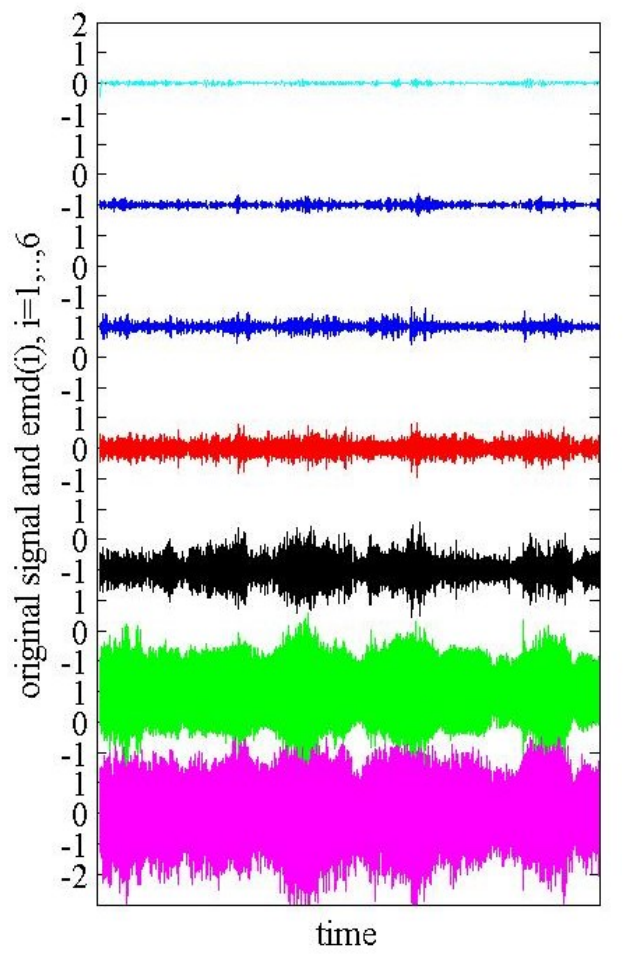

(b)

Fig. 2. Decomposition of the measured acceleration signals (acceleration in the $\mathrm{x}, \mathrm{y}$ and $\mathrm{z}$ directions for (a)-(c), corresponding to Figs. 1(b)-(d), respectively into empirical modes. In the lower panel the original (measured) signal is placed and above in the subsequent higher panels the corresponding modes in the increasing order. It is worth to note that the first two modes have considerably larger amplitude values.

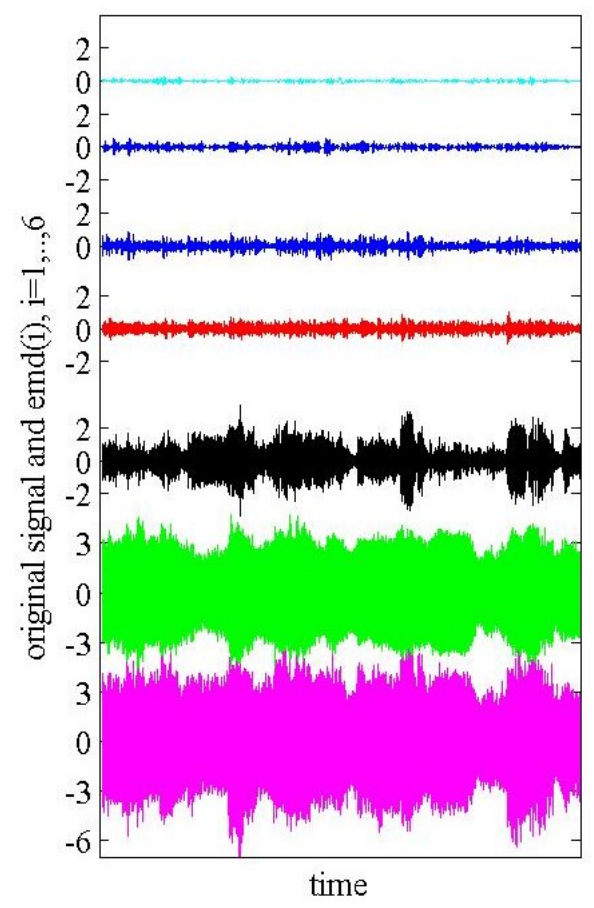

(c)

Fig. 2. Continuation.

In the next step, the Hilbert transform is performed in individual modalities, allowing for the separation of temporal amplitude, phase angle, and frequency. The Hilbert transform is defined from the original signal $g(t)=A_{\mathrm{i}}^{\mathrm{j}}(t)$ as follows:

$$
\hat{g}(t)=\frac{1}{\pi} \int_{-\infty}^{\infty} \frac{g(\tau)}{t-\tau} d \tau .
$$

Defining a new complex function $z(t)$ :

$$
z(t)=g(t)+i \hat{g}(t)
$$

We obtain information about the instantaneous amplitude, A, phase, $\theta$, and frequency, $\mathrm{f}$ :

$$
\begin{aligned}
& A(t)=\sqrt{(g(t))^{2}+(\hat{g}(\iota)),} \\
& \theta(t)=\operatorname{arctg}\left(\frac{\hat{g}(\iota)}{g(t)}\right), f=\frac{1}{2 \pi} \frac{d \theta}{d t} .
\end{aligned}
$$

Table. 1. Standard deviation $\sigma$ of the original signal (measured) and corresponding standard deviations in successive oscillation modes.

\begin{tabular}{|c|c|c|c|c|c|c|c|}
\hline & $\sigma$ & $\begin{array}{c}\text { Mod } \\
1\end{array}$ & $\begin{array}{c}\text { Mod } \\
2\end{array}$ & $\begin{array}{c}\text { Mod } \\
3\end{array}$ & $\begin{array}{c}\text { Mod } \\
4\end{array}$ & $\begin{array}{c}\text { Mod } \\
5\end{array}$ & $\begin{array}{c}\text { Mod } \\
6\end{array}$ \\
\hline $\mathrm{x}$ & 0.653 & 0.748 & 0.646 & 0.235 & 0.176 & 0.108 & 0.051 \\
\hline & 1.020 & 0.925 & 0.344 & 0.180 & 0.122 & 0.080 & 0.040 \\
\hline $\mathrm{z}$ & 2.202 & 0.946 & 0.335 & 0.090 & 0.097 & 0.057 & 0.027 \\
\hline
\end{tabular}


The results of Hilbert-Huang transform (by considering first 6 modes presented in Fig. 2) are summarised in Fig. 3. They show high similarity in frequencies but differs by amplitudes. These tendencies were visible in Figs. 1 and 2.

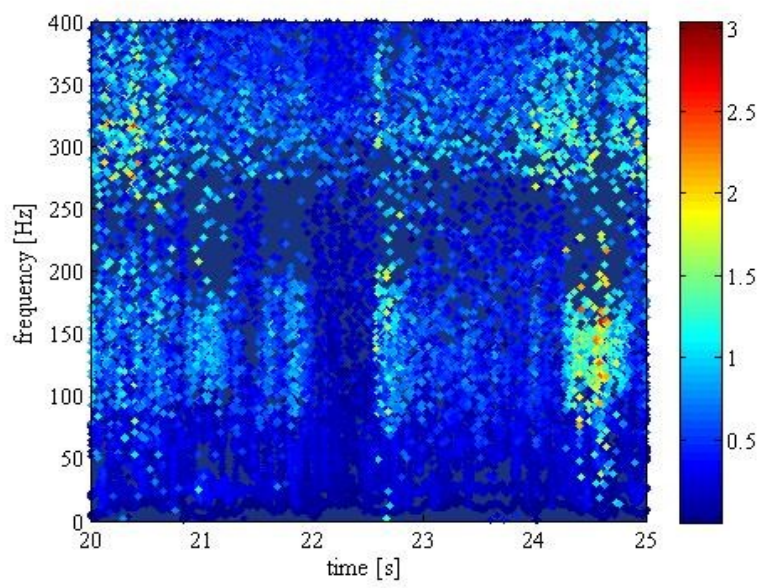

(a)

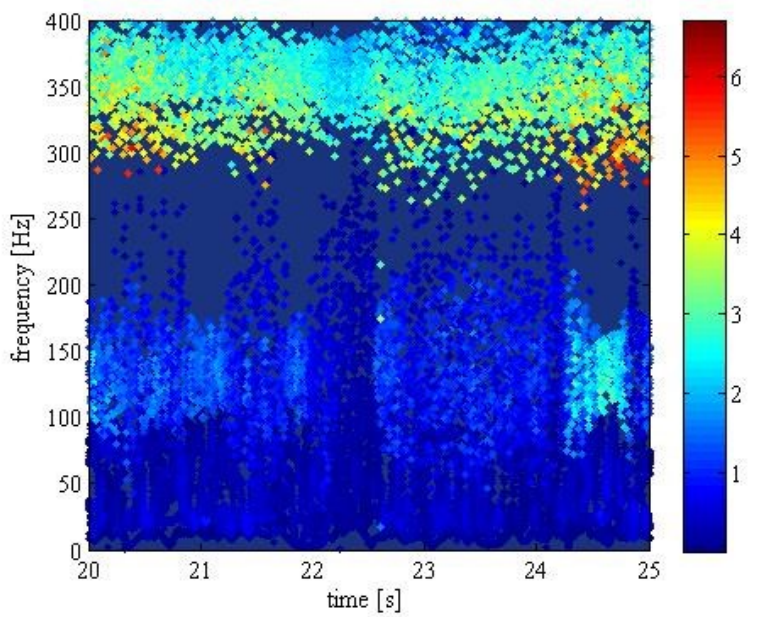

(b)

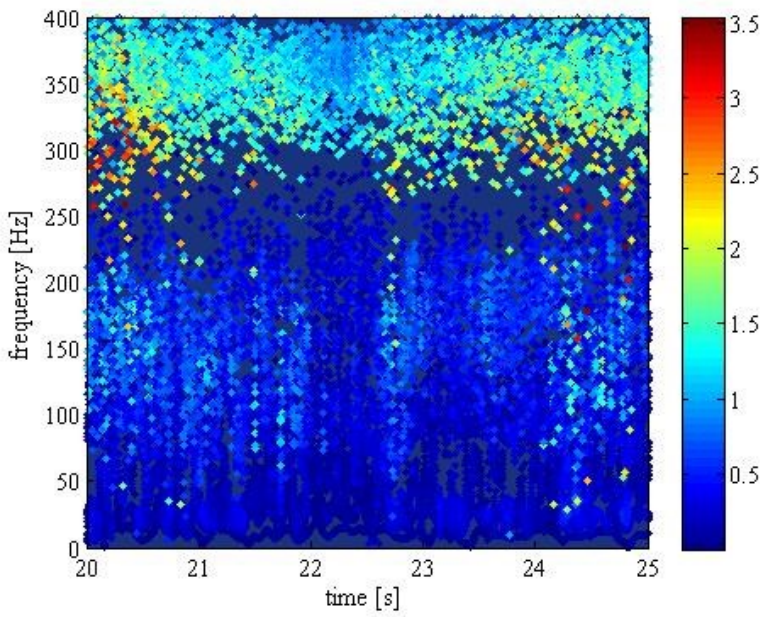

(c)

Fig. 3. Hilbert-Huang results for three considered cases. Colours denote to the amplitudes in a linear scale.
Furthermore, Figs. 4-6 show the distribution of instantaneous frequencies and amplitudes for the directions of $\mathrm{x}, \mathrm{y}$ and $\mathrm{z}$ vibrations from the first four modes (Fig. 2). The resonance back bones matched to the results, indicating that the 2nd and 3rd fashion are non-linear. For these cases, the back-bone curve is clearly skewed and even changes the curvature with amplitude. It should be emphasised that linear systems are characterised by a straight back bone line.

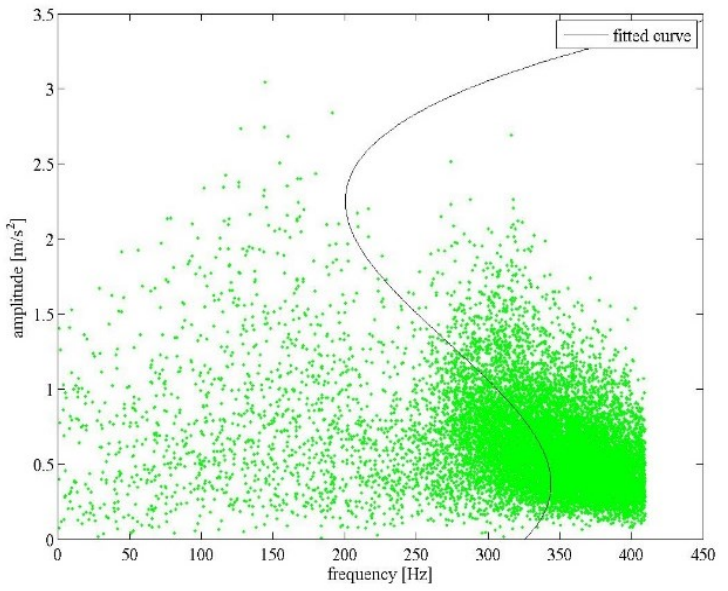

(a)

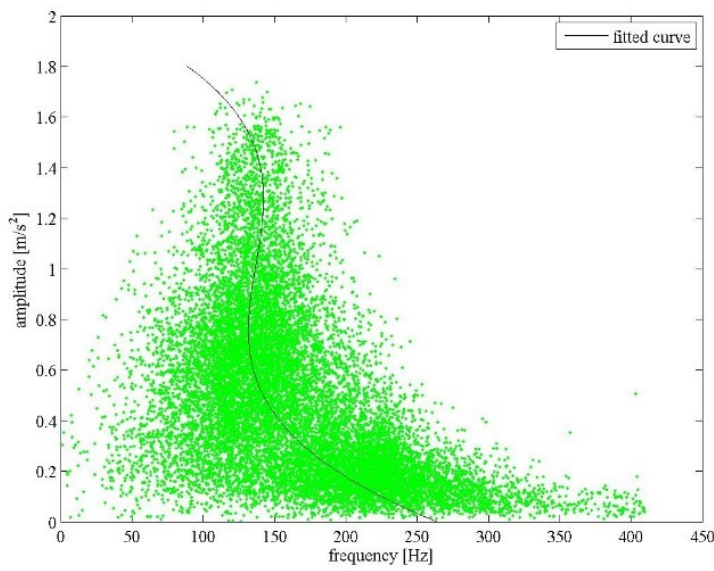

(b)

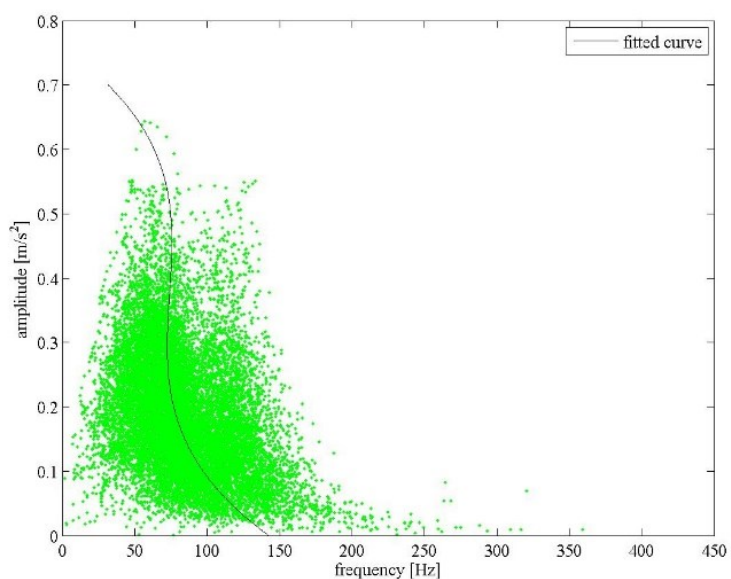

(c) 


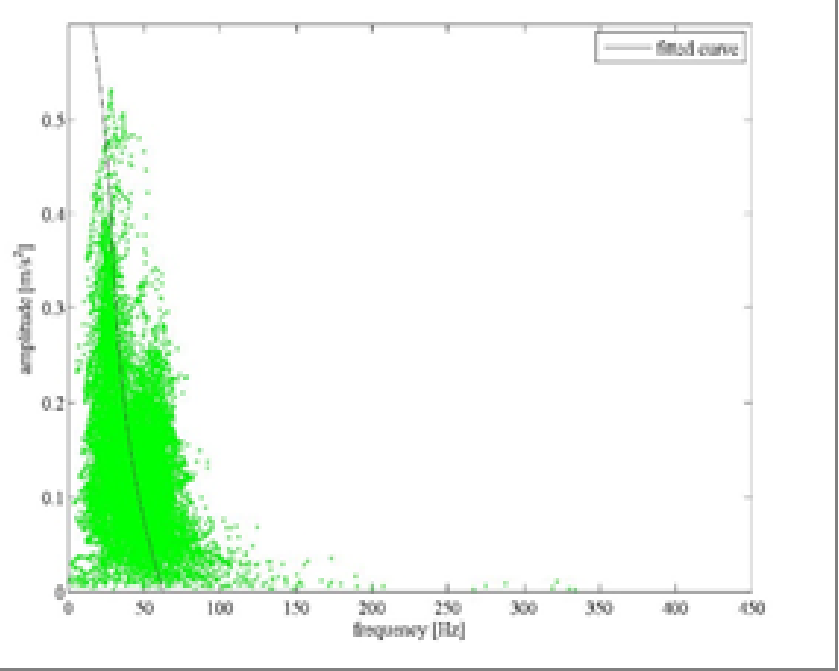

(d)

Fig. 4. Distribution of instantaneous frequencies and amplitudes for the first (a), second (b), third (c), and fourth (d) modes. The signal was measured in the $\mathrm{x}$ direction. The continuous line is a polynomial (third degree polynomial) matching the corresponding back bones of the resonance.

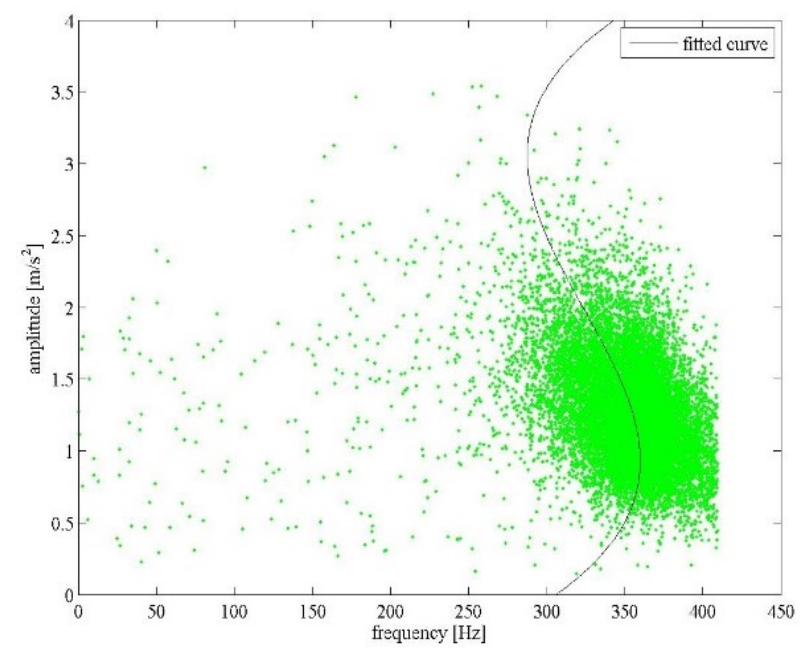

(a)

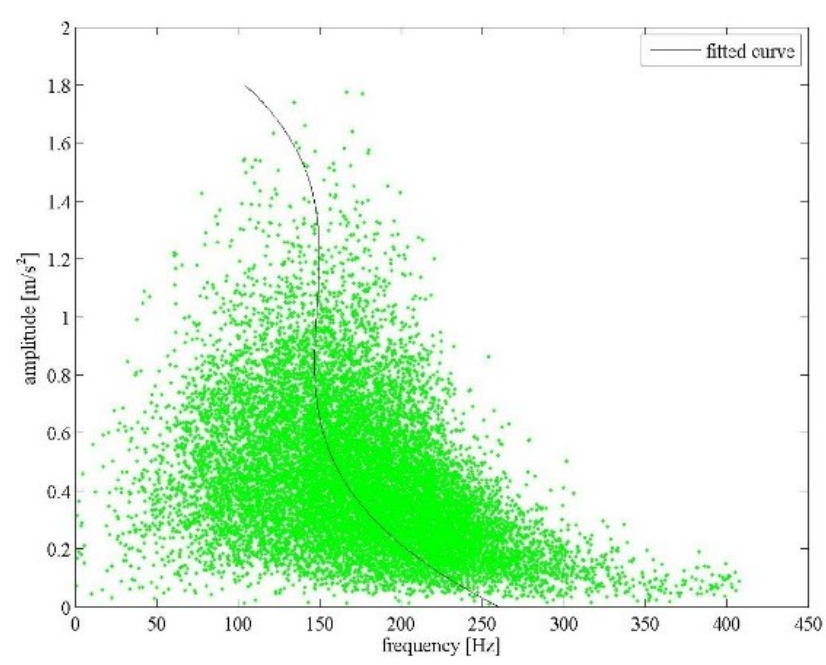

(b)

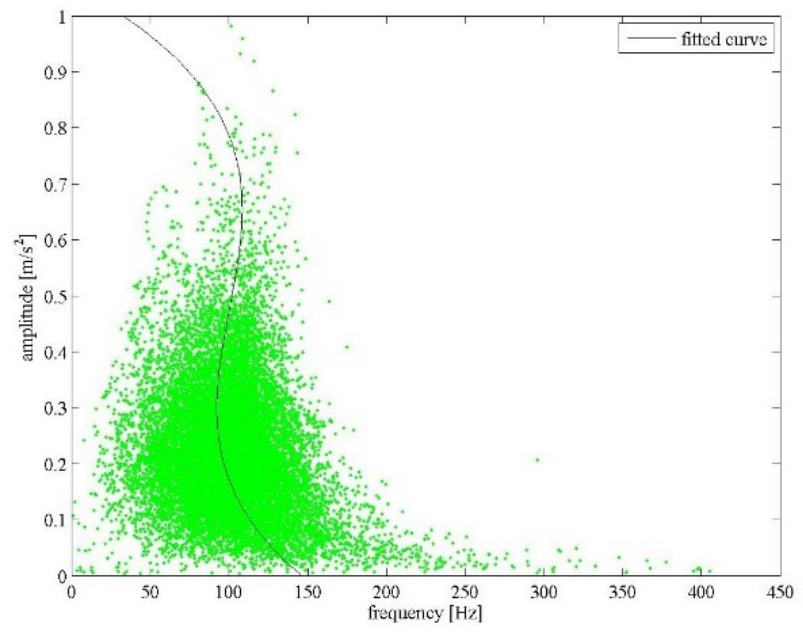

(c)

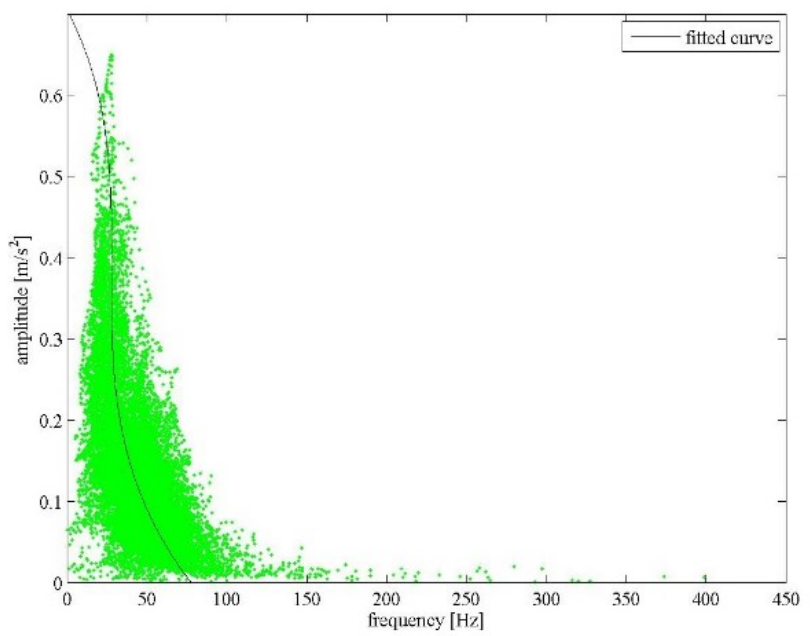

(d)

Fig. 5. Distribution of instantaneous frequencies and amplitudes for the first (a), second (b), third (c), and fourth (d) modes. The signal was measured in y direction. The continuous line is a polynomial (third degree polynomial) matching the corresponding back bones of the resonance.

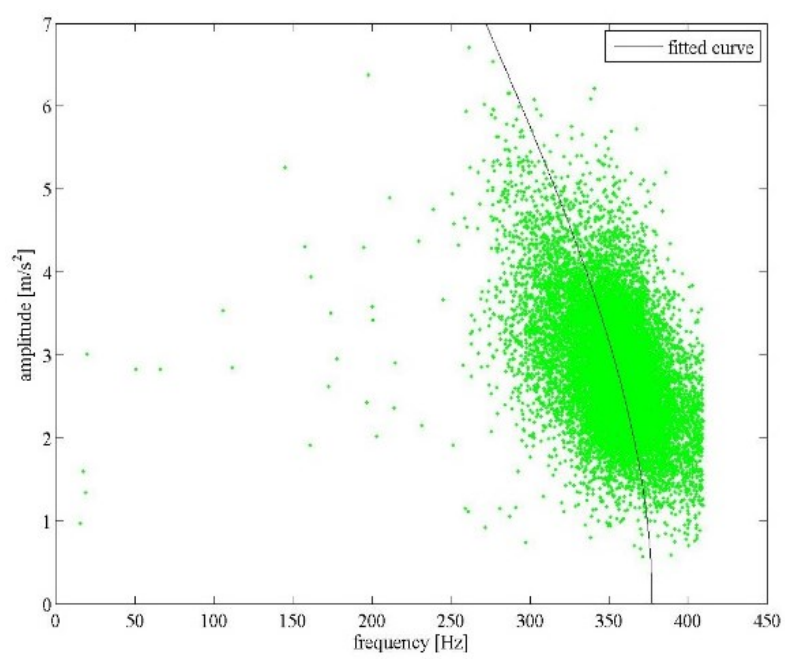

(a) 


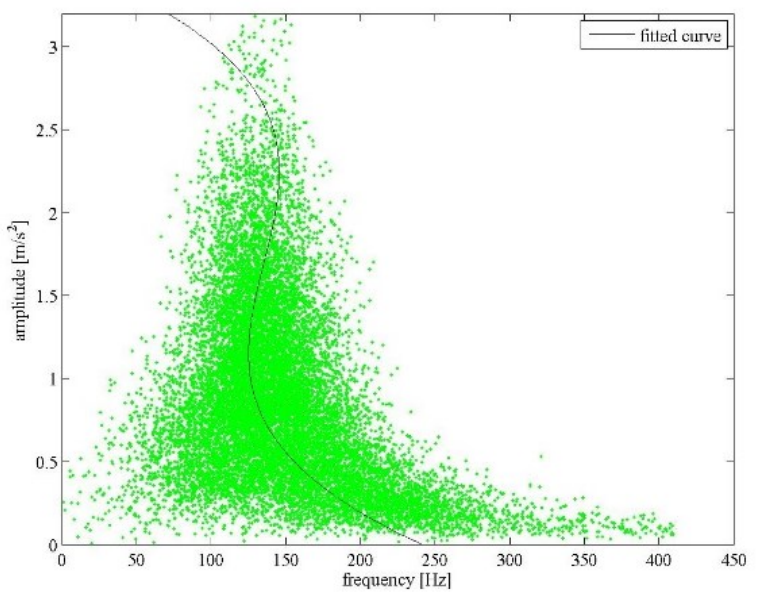

(b)

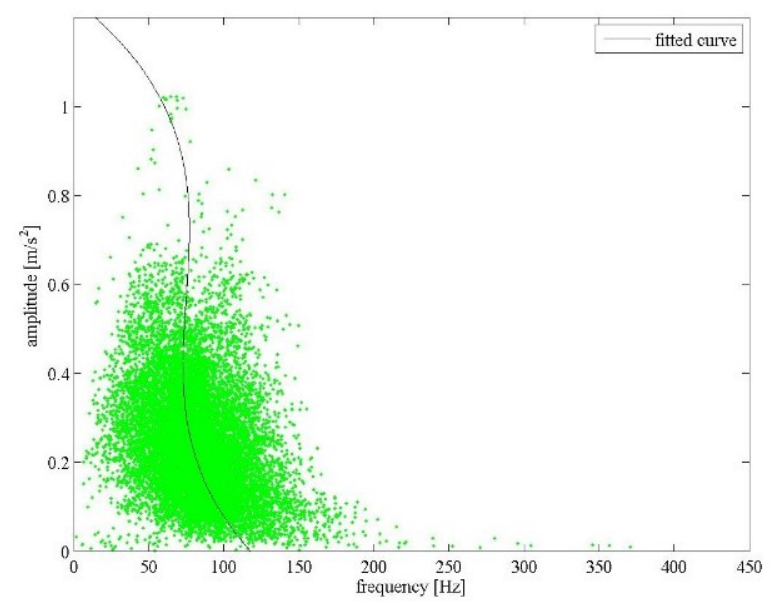

(c)

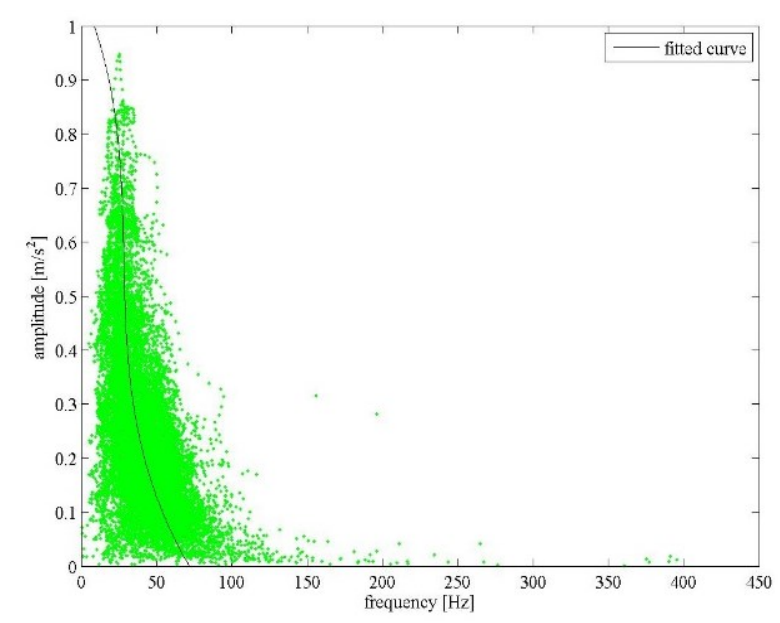

(d)

Fig. 6. Distribution of instantaneous frequencies and amplitudes for the first (a), second (b), third (c) and fourth (d) modes. The signal was measured in $\mathrm{z}$. The continuous line is a polynomial (third degree polynomial) matching the corresponding back bones of resonance.

\section{Conclusions}

The presented analysis includes a number of positive features that can be used in modelling the frame and milling attachment of the milling machine. First, Huang's transformation does not require stationary time. For this purpose, various stages of the milling machine can be used. Secondly, this analysis provides information on nonlinear modal vibrations. Thirdly, the separation of empirical models has a broader meaning and the research technique can be enriched with other elements by creating the original hybrid technique dedicated to particular tasks.

\section{References}

1. F. Al-Bender, W. Symens, J. Swevers, H. Van Brussel, 2004, Int. J. Nonlin. Mech. 39, 1721 (2004)

2. V. Babitsky, V.L Krupenin, Vibrations of strong nonlinear discontinuous systems, (Springer, Berlin, 2001)

3. G. Kerschen, K. Worden., A.F. Vakakis, J.C. Golinval, Mech. Systems and Sign. Process. 20, 505 (2006).

4. A.H. Nayfeh L. Pai, Linear and nonlinear structural Mechanics (Wiley Interscience, New York, 2004)

5. M. Haroon, D.E. Adams., Y.W. Luk, ASME J. Vibr. Acoust., 127, 483 (2005).

6. J. Iwaniec, Key Engineering Materials, 347, 467 (2007)

7. J. Iwaniec, M. Iwaniec, Solid State Phenomena, 248, 175 (2016).

8. D. Hilbert, Grundzuege einer allgemeinen Theorie der linearen Integralgleichungen, (Chelsea Pub. Co., New York, 1953)

9. N.E. Huang, Z. Shen, S.R. Long, M.L.C Wu., H.H. Shih, Q.N. Zheng, N.C. Yen, C.C. Tung, H.H. Liu, Proc. Roy. Soc. London A454, 903 (1998)

10. P. Wolszczak, K. Lygas, G. Litak, Advances in Science and Technology Research Journal 11, 96 (2017)

11. G. Litak, K. Kecik, R. Rusinek, Latin Amer. J. Solids Struct. 10, 133 (2013) 